\title{
Multi-Epoch VLBI observations of the extreme scintillator $\mathrm{J} 1819+3845$
}

\section{Giuseppe Cimò and Leonid Gurvits}

\author{
Joint Institute for VLBI in Europe, \\ Postbus 2, NL-9700TA, Dwingeloo, the Netherlands \\ email: cimo@jive.nl
}

\begin{abstract}
The quasar J1819+3845 has shown extreme variability with flux density variations in the radio regime up to $600 \%$ in less than one hour. In case of intrinsic high varibility, the short time scale sets a limit on the size of the emitting region and allows to estimate its brightness temperature. This would exceed $10^{21} \mathrm{~K}$ in the case of J1819+3845. Even an high relativistic jet beamed and doppler boosted in our line of sight cannot explain such an extreme violation of the Inverse Compton limit $\left(10^{12} \mathrm{~K}\right)$. The variability of this source has been proven to be due to scattering in the Interstellar medium by a number of different experiments. Such an explanation requires a closeby scattering screen (few parsecs) and it results in a brightness temperature of about $10^{14} \mathrm{~K}$. Many observing campaigns have been carried on to map the innermost jet structures of J1819+3845. Here we present the results of a number of VLBI observations, including space VLBI, to search for the missing jet in this puzzling source.
\end{abstract}

Keywords. Scintillation, VLBI, jets

\section{Introduction}

Intra-Day Variable sources (IDV's) are flat spectrum extragalactic synchrotron sources, often associated with optical quasars, BL Lac's or radio galaxies, that show flux density variability on time scales shorter than a day (Witzel et al. 1986 and Heeschen et al. 1987). These sources show also intrinsic variations on much longer time scales (months) that sometimes are associated with milli-arc-second structure that evolves on time scales of years. On the other hand, the intra-day variations are easily explained as a propagation effect due to scintillation in an interstellar screen of ionized gas (Rickett et al. 2001 and references therein).

The quasar J1819+3845 has shown extreme variability with flux density variations in the radio regime up to $600 \%$ in less than one hour (Dennett-Thorpe \& De Bruyn 2000). In case of intrinsic high variability, the short time scale sets a limit on the size of the emitting region and allows one to estimate its brightness temperature. The latter would exceed $10^{21} \mathrm{~K}$. Even a highly relativistic jet beamed and doppler boosted toward the line of sight cannot explain such an extreme violation of the Inverse Compton limit $\left(10^{12} \mathrm{~K}\right)$. The variability of this source has been proven to be due to scattering in the Interstellar medium by a number of different experiments. Such an explanation requires a closeby scattering screen (few parsecs, Dennett-Thorpe \& De Bruyn 2002 and Dennett-Thorpe \& De Bruyn 2003) and it results in a brightness temperature of about $10^{14} \mathrm{~K}$, still exceeding the inverse Compton limit and implying a very compact structure.

A number of VLBI campaigns have been carried out to image the innermost jet structures of J1819+3845. Three global VLBI experiments, including a Space VLBI epoch, and two observing sessions with the VLBA and the EVN arrays. However the main issue with imaging $\mathrm{J} 1819+3845$ has been its extreme variability. The data reduction is 
compromised by the difficulties in dealing with phase fluctuations and scintillation. Furthermore, the interstellar scintillation induced variations will cover the structure of the source due to the scattering broadening of the VLBI image.

\section{The variability and the jet structure}

The extreme variability of J1819+3548 has been observed since its first observation in 1999 and it has continued for more than 8 years. Due to its high brightness temperature and the compact nature, J1819+3845 was expected to expand quenching the scintillation or at least changing its variability characteristics following its internal structural variations. However, the variations have been observed continuously along the years showing also a clear annual modulation due to relative motion of the Earth and the scattering screen.

On one hand, the scintillation has prevented to image the milli-arcsecond structure of this puzzling object. On the other hand, it has been a powerful tool to study indirectly the source structure at $\mu$-arcsecond scale. Macquart \& de Bruyn (2007) have compared observations taken at identical epochs each year in order to disentangle the effects of source structural evolution and asymmetry in the scintillation pattern. Analysing the light curves and their power spectra, it was possible to indicate the evolution of the internal structure of J1829+3845. Comparing the scintillation characteristics in 2003, 2004 and 2006, Macquart \& de Bruyn (2007) found an expanding double structure.

In 2008, EVN observations of J1819+3548 (Cimò et al. 2008) have shown no signs of any variability (see attached plots to compare the extreme scintillation to the quiescent phase). A possible explanation for the cessation of the fast variations in J1819+3548 is that the scattering screen has moved away from our line of sight. On the other hand, the disappearance of the extreme scintillations on J1819+3845 could be due to variations in the source structure at $\mu$-arcsecond scale. A new component, which expands as it evolves would lead to episodic scintillations. The EVN data have shown however that the source is unresolved at the European baselines. We have analized the previous VLBI observations of J1819+3548 and we have not found a confirmation for any structural changes with time.

However our imaging process has been so far limited by the scintillation. A model of the source in its current quiescent phase is in progress. This will allow us to retroactively interpret the scintillation-limited data and can provide us with information on the structure of the innermost jet structures of the source and their evolution in the course of the previous 10 years.

\section{References}

Cimò, G. 2008, PoS(IX EVN Symposium), 13

Dennett-Thorpe, J. \& De Bruyn, A. G. 2000, ApJ, 529, L65

Dennett-Thorpe, J. \& De Bruyn, A. G. 2001, Nature, 415, 57

Dennett-Thorpe, J., De Bruyn, A. G. 2003, A\&A, 404, 113

Heeschen, D. S., Krichbaum, T., Schalinski, C. J., \& Witzel, A. 1987, AJ, 94, 1493

Macquart, J.-P. \& De Bruyn, A. G. 2007, MNRAS, 380, L20

Rickett, B. J., Witzel, A., Kraus, A., Krichbaum, T. P., \& Qian, S. J. 2001, ApJ, 550, L11

Witzel, A., Heeschen, D. S., Schalinski, C., \& Krichbaum, T. 1986 Mitteilungen der Astronomischen Gesellschaft Hamburg, 65, 239 